\title{
Laparoscopic Hepatectomy is Easier in Repeat Hepatectomy
}

\author{
Naruhiko Ikoma, MD, Osamu Itano, MD, PhD, Go Oshima, MD, Shinil K. Shah, MD, \\ Shinichi Fukuhara, MD, Yuko Kitagawa, MD, PhD, FACS
}

Department of Surgery, University of Texas Medical School at Houston, Houston, Texas, USA (Drs. Ikoma and Shah); Keio University, School of Medicine, Tokyo, Japan (Drs. Ikoma, Itano, Oshima, and Kitagawa); Department of Surgery, Eiju General Hospital, Tokyo, Japan (Drs. Ikoma, Itano, and Oshima); Department of Surgery, Beth Israel Medical Center, New York, New York, USA (Dr. Fukuhara); Michael E. DeBakey Institute for Comparative Cardiovascular Science and Biomedical Devices, Texas A\&M University, College Station, Texas, USA (Dr. Shah).

\begin{abstract}
Hepatic malignancies are frequently associated with primary liver disease, including hepatitis and cirrhosis. It is not uncommon for selected patients to require repeated operations for tumor recurrence. Subsequent operations are more challenging because of dense adhesions from prior operations combined with the inherent risk for liver dysfunction. We present the case of a 64-year-old man with a 20-year history of hepatitis B who developed recurrent hepatocellular carcinoma and underwent repeated laparoscopic hepatectomies on 4 separate occasions. We found that the laparoscopic approach in repeat hepatectomy offers significant advantages, including minimal adhesions, superior visualization, and favorable effect by generated by pneumoperitoneum. Laparoscopic hepatectomy will potentially become a standard strategy for the treatment of liver malignancies associated with background liver abnormalities, which carry the high possibility of reemergence of the liver malignancy in the remnant of the liver.
\end{abstract}

Key Words: Laparoscopic surgery, Hepatectomy, HCC, Hepatitis.

Citation Ikoma N, Itano O, Oshima G, Shah SK, Fukuhara S, Kitagawa Y. Is Laparoscopic Hepatectomy easier in repeat hepatectomy? CRSLS e2014.00202. DOI: 10.4293/CRSLS.2014.00202.

Copyright (C) 2015 by SLS, Society of Laparoendoscopic Surgeons. This is an open-access article distributed under the terms of the Creative Commons Attribution-Noncommercial-ShareAlike 3.0 Unported license, which permits unrestricted noncommercial use, distribution, and reproduction in any medium, provided the original author and source are credited.

Address correspondence to: Osamu Itano, MD, PhD, Department of Surgery, Keio University, School of Medicine, 35 Shinanomachi, Shinjuku-ku, Tokyo 160-8582, Japan, Tel: 81-3-3353-1211, Fax: 81-3-3355-4707, E-mail: itano@z8.keio.jp

\section{INTRODUCTION}

Patients who develop hepatocellular carcinoma (HCC) commonly have background liver abnormalities such as hepatitis and cirrhosis. Liver transplantation is commonly indicated for such patients, but limitations exist ${ }^{1}$; therefore, surgical resection remains the main option for treatment of localized HCC. ${ }^{1}$ However, the high frequency of reemergence of HCC in the remnant of the diseased liver makes the treatment challenging. Studies have shown that repeat tumor resection of recurrent HCC improved survival compared with other treatment modalities, ${ }^{1-5}$ and long-term results were as favorable as salvage liver transplantation. ${ }^{6}$ The current consensus is that repeat hepatectomy of recurrent HCC offers the best chance for cure and should be considered whenever possible.1,3 However, open hepatectomy generally requires a large incision ac- companied by significant invasiveness, which may limit the ability to perform repeat surgery. Although favorable outcomes after second or third hepatectomies have been reported, surgeons uniformly report that repeat hepatectomy is technically more demanding than primary resection. ${ }^{1-3}$ We present a case of 4 sequential laparoscopic hepatectomies for reemergence of HCC in a patient with chronic hepatitis B. The surgical procedures were uncomplicated, and we found that the laparoscopic approach offers a number of favorable characteristics during repeat hepatectomies.

\section{CASE DESCRIPTION}

The presented patient is a 64-year-old man with a 20-year history of hepatitis B who was found to have a 2.5 -cm liver tumor in segment 6 on screening computed tomography 


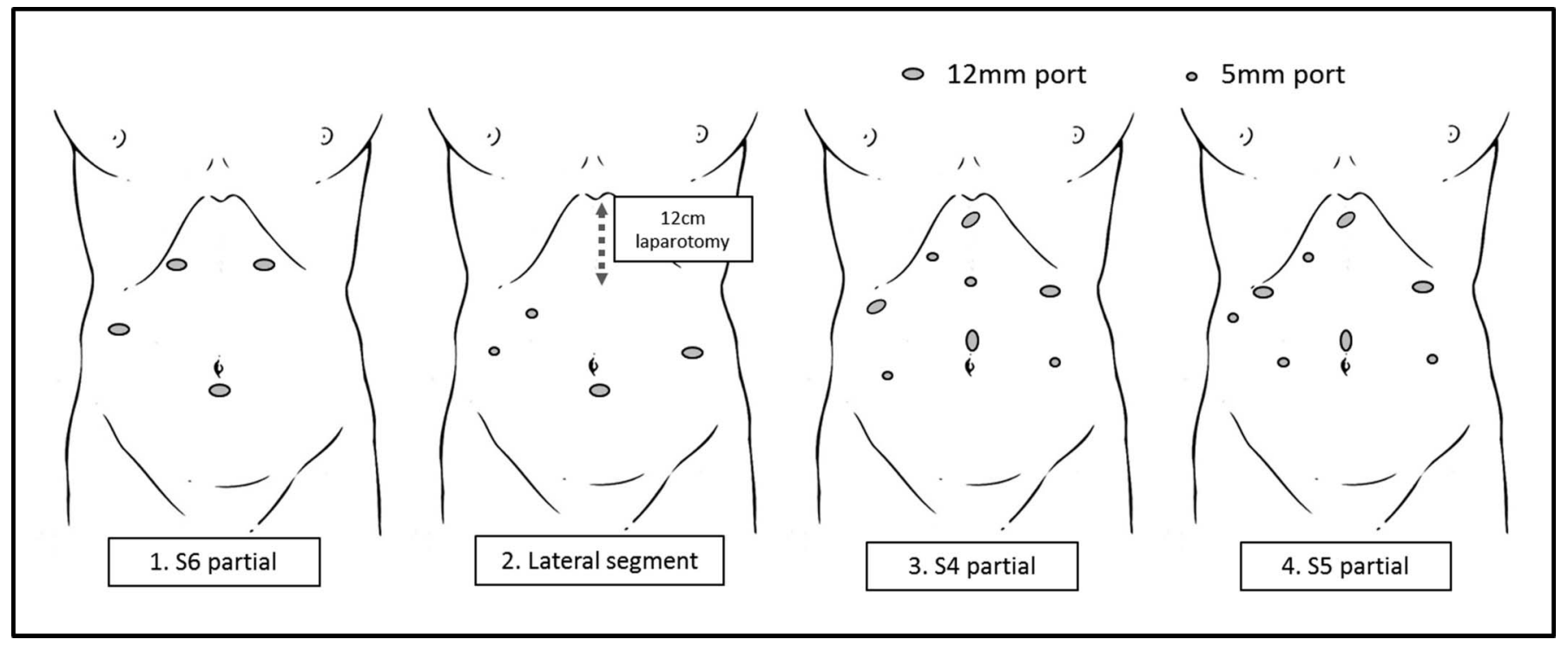

Figure 1. Port placement for each of the 4 operations: (1) S6 partial hepatectomy, (2) lateral segmentectomy, (3) S4 partial hepatectomy, and (4) S5 partial hepatectomy.

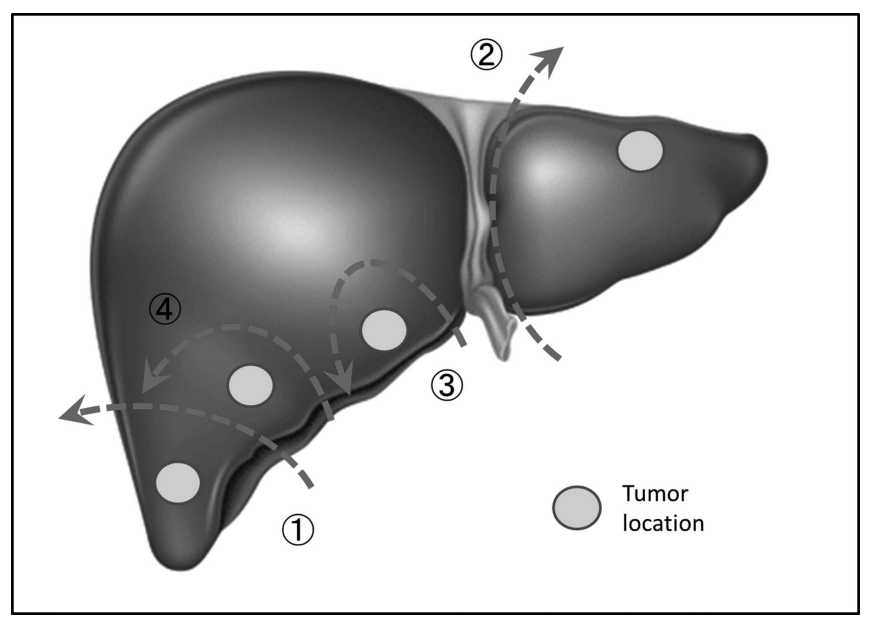

Figure 2. Diagram describing location of initial tumor and recurrences: (1) S6 partial hepatectomy, (2) lateral segmentectomy, (3) S4 partial hepatectomy, and (4) S5 partial hepatectomy.

and subsequent magnetic resonance imaging, for which he underwent a laparoscopic partial hepatectomy. He subsequently developed recurrent HCC in segments 2 (2 $\mathrm{cm}), 4(1.5 \mathrm{~cm})$, and $5(2 \mathrm{~cm})$ at $2,3.3$, and 4 years, respectively, after the first operation. Each recurrence was treated with repeated laparoscopic hepatectomy (Figures 1, 2, and 3). Operative time, estimated blood loss, length of stay after each surgery, and complications are shown in Table 1. Of note, the patient developed a perforated duodenal ulcer after the third hepatectomy, for which he underwent a laparoscopic Graham patch procedure on postoperative day 5 after the third hepatectomy, with an otherwise unremarkable postoperative course. Pathology revealed HCC in all 4 specimens arising from a background of cirrhosis. There was no evidence of resectionmargin recurrence after any of the hepatectomies, and the recurrences were thought to be secondary to reemergences from his hepatitis B and cirrhosis. After the fourth hepatectomy, he developed small multiple recurrences in segments 4, 5, and 6 and subsequently underwent transcatheter arterial chemoembolization. Now, 7 years after the first hepatectomy, he remains alive with controlled disease and maintained liver function.

\section{DISCUSSION}

In addition to its minimal invasiveness, there are 3 reasons that laparoscopic hepatectomy will potentially become a standard strategy for the treatment of recurrent liver malignancies. First, laparoscopic hepatectomy creates minimal intra-abdominal adhesions. Posthepatectomy adhesions can be divided into 2 categories: adhesions on the transected surface of the liver (transection adhesions) and adhesions underneath the laparotomy incision (incisional adhesions). Open hepatectomy creates large incisional adhesions atop the liver, and reopening the same incision may be difficult. The laparoscopic approach to hepatectomy minimizes incisional adhesions. Repeated laparoscopic hepatectomy usually starts from an adhesion-free area in the middle to lower abdomen, and surgeons can usually easily 


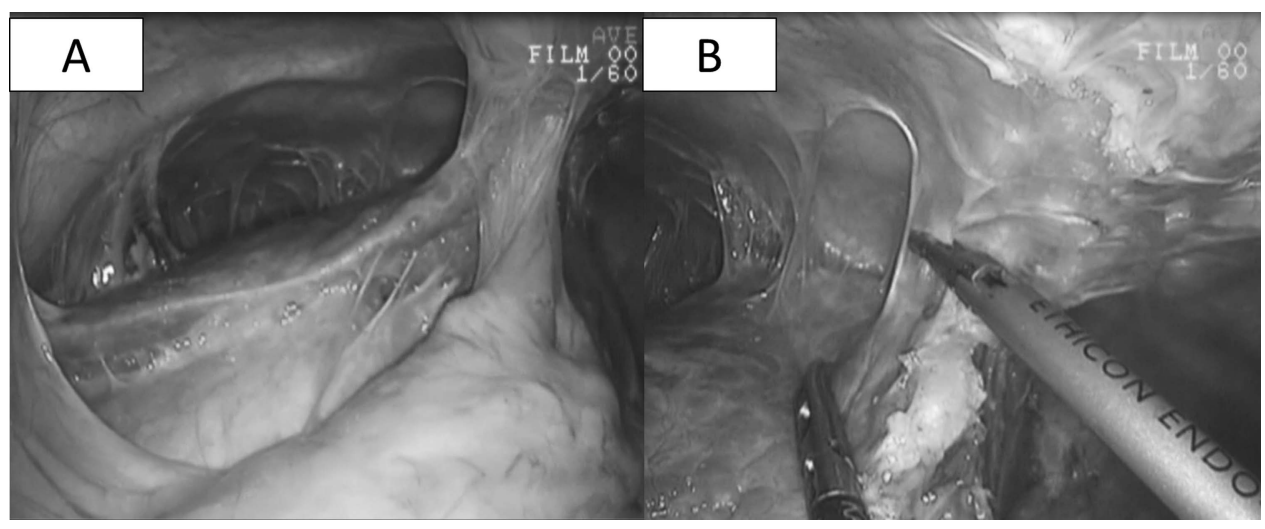

Figure 3. Operative pictures from the third resection ( $\mathrm{S} 4$ partial hepatectomy): (A) Showing incisional adhesions under the previous laparotomy. The space created by pneumoperitoneum makes dissection of the adhesions easier. (B) Pneumodissection dissects the weak adhesions and directs the surgeon along the current dissection plane. Clear visualization provides safe and precise adhesiolysis.

Table 1.

Surgery Outcomes

\begin{tabular}{|c|c|c|c|c|}
\hline Variable & First Hepatectomy & Second Hepatectomy & Third Hepatectomy & Fourth Hepatectomy \\
\hline Location & S6 & S2 & S4 & S5 \\
\hline Size $(\mathrm{mm})$ & 25 & 20 & 15 & 20 \\
\hline Procedure & Partial hepatectomy & Lateral segmentectomy & Partial hepatectomy & Partial hepatectomy \\
\hline OR time (min) & 386 & 188 & 250 & 250 \\
\hline $\mathrm{EBL}(\mathrm{mL})$ & 150 & 200 & 5 & 200 \\
\hline $\operatorname{LOS}(d)$ & 10 & 7 & 13 & 12 \\
\hline Complications & - & - & $\begin{array}{l}\text { Perforation of } \\
\text { duodenal ulcer }\end{array}$ & - \\
\hline Pathology & $\begin{array}{l}\text { Moderately } \\
\text { differentiated HCC }\end{array}$ & $\begin{array}{l}\text { Moderately } \\
\text { differentiated HCC }\end{array}$ & $\begin{array}{l}\text { Moderately } \\
\text { differentiated HCC }\end{array}$ & $\begin{array}{l}\text { Moderately } \\
\text { differentiated HCC }\end{array}$ \\
\hline
\end{tabular}

EBL, estimated blood loss; HCC, hepatocellular carcinoma; LOS, length of stay; OR, operating room.

create a working space with minimal difficulty. We performed minilaparotomy for the second operation, which was a lateral segmentectomy. This could be completed with purely laparoscopic technique, but we felt it was more reasonable to make a small incision, which was required for specimen extraction anyway, to perform liver transection safely. The downside of the minilaparotomy was that it created incisional adhesions. Lysis of adhesions was required at the next operation, which was still feasible with the laparoscopic technique.

Second, laparoscopic hepatectomy can provide superior visualization, especially in the upper abdominal cavity. Laparoscopy provides perpendicular vision against the adhesion between the abdominal wall and the liver, which gives surgeons a better orientation to the liver's shape (Figure 3). Subsequently, lysis of the adhesions can be performed tangential to the liver surface, which minimizes the chance of parenchymal injury.

Last, pneumoperitoneum has favorable effects: pneumodissection and pneumoastriction. The pressure generated by the pneumoperitoneum, which is usually set at 15 $\mathrm{mm} \mathrm{Hg}$, pushes apart spaces to expand them. Once the toughest part of the adhesion is transected, this pneumodissection dissects the weaker adhesions behind it and directs the surgeon along the current dissection plane. After lysis of adhesions, the key point of liver parenchymal dissection is bloodless surgery. We use the superficial precoagulation sealing and transection technique, ${ }^{7}$ which uses meticulous precoagulation with VIO Soft-Coagulation to minimize bleeding. The pressure by pneumoperitoneum also facilitates hemostasis during liver transection. 
This pneumoastriction helps control venous oozing and provides the surgeon with clear vision, helping facilitate a safe procedure.

We believe this case report shows the potential benefit of the laparoscopic technique in repeat hepatectomies, but patient selection is important. We believe the ideal candidates for repeat laparoscopic hepatectomy are patients with peripherally located small tumors, because they require minimal liver mobilization and thus create fewer adhesions, but tumors that require anatomic resection are still not contraindicated for the laparoscopic technique with experienced surgeons. Because the tissue reaction after the liver resection and the degree of adhesion formation have significant individual variation, we recommend starting with diagnostic laparoscopy and judicious conversion to open surgery to provide safety.

\section{CONCLUSIONS}

Minimally invasive laparoscopic hepatectomy for malignancy may provide good local control equivalent to that of open hepatectomy in selected patients. The laparoscopic approach in repeat hepatectomy may offer significant advantages, including minimal adhesions, superior vision, and favorable effects generated by pneumoperitoneum. Laparoscopic hepatectomy will potentially become a standard strategy for the treatment of liver malignancies associated with background liver abnormalities, which carry the high possibility of reemergence of the liver malignancy in the remnant of the liver.

\section{References:}

1. Wu CC, Cheng SB, Yeh DC, Wang J, P'Eng FK. Second and third hepatectomies for recurrent hepatocellular carcinoma are justified. Br J Surg. 2009;96:1049-1057.

2. Nakajima $Y$, Ko S, Kanamura T, et al. Repeat liver resection for hepatocellular carcinoma. J Am Coll Surg. 2001;192:339-344.

3. Tralhao JG, Dagher I, Lino T, Roudie J, Franco D. Treatment of tumour recurrence after resection of hepatocellular carcinoma. Analysis of 97 consecutive patients. Eur J Surg Oncol. 2007;33:746-751.

4. Faber W, Seehofer D, Neuhaus P, et al. Repeated liver resection for recurrent hepatocellular carcinoma. J Gastroenterol Hepatol. 2011;26:1189-1194.

5. Kishi Y, Saiura A, Yamamoto J, et al. Repeat treatment for recurrent hepatocellular carcinoma: is it validated? Langenbecks Arch Surg. 2011;396:1093-1100.

6. Ng KK, Lo CM, Liu CL, Poon RT, Chan SC, Fan ST. Survival analysis of patients with transplantable recurrent hepatocellular carcinoma: implications for salvage liver transplant. Arch Surg. 2008;143:68-74.

7. Itano O, Ikoma N, Takei H, Oshima G, Kitagawa Y. the superficial precoagulation, sealing, and transection method: a "bloodless" and "ecofriendly" laparoscopic liver transection technique. Surg Laparosc Endosc Percutan Tech. In press. 